\title{
Granular configurations, motions, and correlations in slow uniform flows driven by an inclined conveyor belt
}

\begin{abstract}
The present experimental study examines the behaviour of slow granular flows, focusing on the details of particle patterns and motions over the depth of a sheared layer. A conveyor belt circuit enclosed in an inclined flume is used to generate steady uniform open-channel flows of dry granules. Particle positions near the transparent sidewall are extracted from video sequences. The Voronoï diagram is then used to characterise the configurations formed by neighbouring grains and to assist particle tracking over successive frames. This allows a qualitative visualisation of the internal structure of the flowing layer, as well as quantitative measurements of lattice defect density and granular velocities at different depths. The response of the depth profiles to different conveyor belt speeds is examined. In addition to the mean and fluctuating velocities, we probe the time and space correlations of the fluctuations.
\end{abstract}

\section{Introduction}

Dry granular flows are encountered in a variety of geophysical phenomena and industrial processes. Apart from applications, they are of interest as a striking example of complex system, for which complicated behaviours arise from the interaction of simple constituents. One of the key complications is the existence of two distinct flow regimes. In rapid granular flows, particles interact through short-lived collisions and the stresses vary roughly as the square of the

\section{A. T. H. Perng}

Graduate Institute of Civil Engineering, National Taiwan University, Taiwan. Former affiliation: Department of Civil Engineering,

National Central University, Taiwan

\section{H. Capart $(\varangle)$}

Department of Civil Engineering and Hydrotech Research Institute, National Taiwan University, Taiwan

H. T. Chou

Department of Civil Engineering,

National Central University, Taiwan shear rate. Slow granular flows, by contrast, are characterised by long-lasting, frictional contacts between grains, and exhibit stress magnitudes which are independent of the rate of deformation. How do microscopic features at the granular scale affect the macroscopic flow behaviour ? For rapid granular flows, convincing answers to this question have emerged from theoretical, computational and experimental studies [1, 2]. Likewise, an understanding of what controls the transition between frictional and collisional behaviour is being reached $[3,4]$. For the slow granular flow regime itself, by contrast, a coherent picture is yet to be assembled. While theoretical approaches have been proposed $[5,6]$, wide gaps remain to be filled using empirical information.

We document in the present paper some detailed experimental observations which we hope can be useful in this regard. They pertain to slow free surface flows of spherical particles examined in an inclined conveyor belt channel. Devices of similar design have been used by previous researchers to study liquid-granular debris flows $[7,8]$. The apparatus is found in the present work to be useful for the study of dry granular flows as well. In particular, conditions very close to steady uniform rectilinear flow are obtained. These conditions are more difficult to approximate in rotating drums [9], annular shear cells [10], or non-recirculating chutes [11], which are the devices most commonly used to study granular shear flows.

To characterise the parallel shear flows, we rely on video images of the grains moving near the sidewall of the channel. Thus a limitation of the present experiments is that while the granular flow is three-dimensional (as opposed to a twodimensional system of particles held between parallel plates), visual access is restricted to the near-wall grains. To analyse the configurations and motions of the visible grains, standard image analysis techniques are complemented by special tools based on the Voronoï diagram [12]. This geometrical construction has been found useful by a number of investigators seeking to analyse experimental or computational systems of interacting particles [13-16]. Here we use the Voronoï diagram for two different tasks: first, to characterise arrangements of neighbouring grains on still images, and secondly 


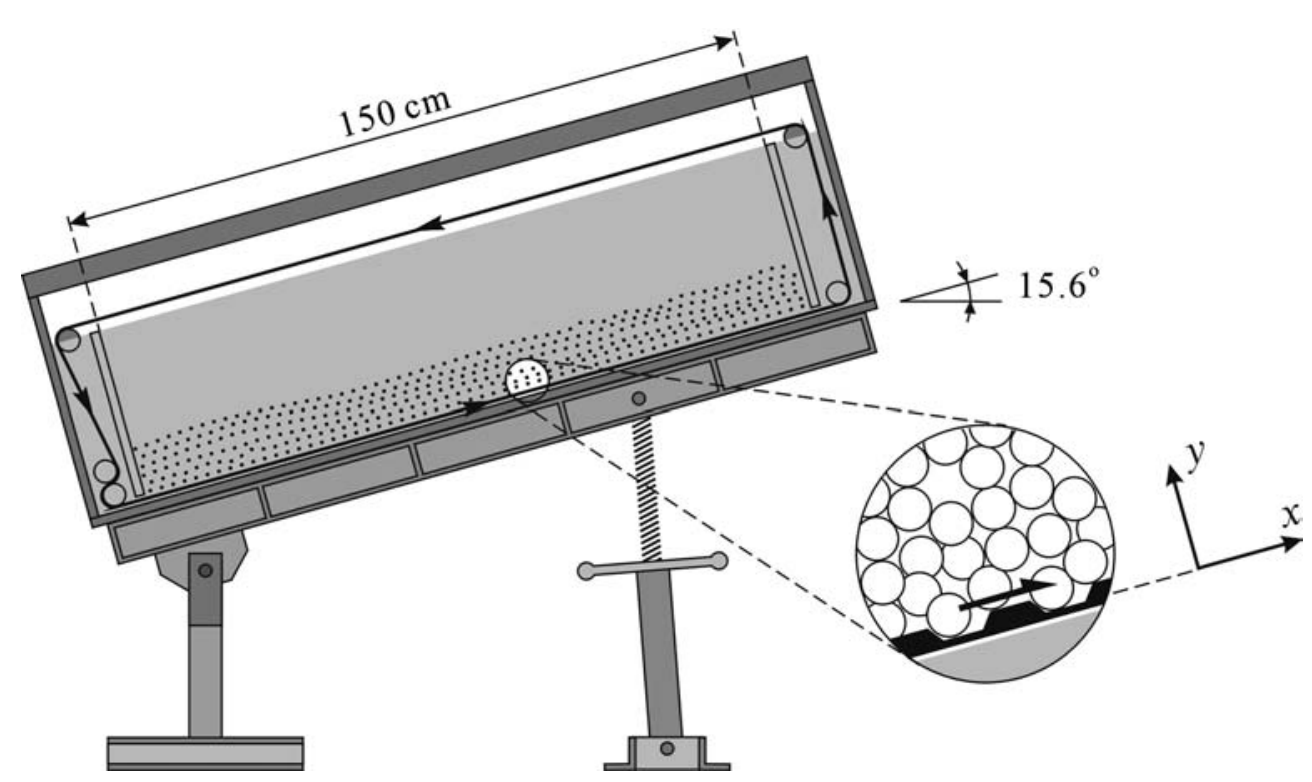

Fig. 1 Inclined channel with internal conveyor belt circuit used for the experiments

to assist velocimetric particle tracking over a sequence of frames.

The paper is organised as follows. Sections 2 and 3 describe the experimental apparatus and imaging techniques, respectively. Section 4 provides a qualitative picture of the internal structure observed in a typical run. Depth profiles of lattice defect densities are used to characterise particle configurations in section 5. Depth profiles of mean and fluctuation velocities are then examined in section 6 . The corresponding time and space correlations are probed in section 7 . Finally, conclusions are drawn in section 8.

\section{Laboratory apparatus and experimental procedure}

Experiments were conducted at the Environmental Fluid Mechanics Laboratory (EFML) of the Department of Civil Engineering, National Central University. The laboratory device adopted for the present research is schematised on Figure 1. A conveyor belt circuit is enclosed in a glass-walled channel of rectangular cross section and adjustable slope. The rubber conveyor belt is mounted on four fixed corner rollers, with a fifth roller used to tune the belt tension. The lower left roller is driven by a variable speed electric motor. The conveyor belt slides along the channel floor in the central compartment of the channel. The vertical plates bounding this compartment left and right have small gaps at the bottom allowing passage of the belt. The inside dimensions of the central compartment are: length $=150 \mathrm{~cm}$; width $=12$ $\mathrm{cm}$; height of the glass sidewalls $=35 \mathrm{~cm}$. The entire device can be inclined at angles going from 0 to 30 degrees. The conveyor belt has transversal grooves of trapezoidal shape (trapeze height $=2.2 \mathrm{~mm}$, base length $=9.2 \mathrm{~mm}$ and top length $=5.8 \mathrm{~mm}$ ), as shown on Figure 1 (inset).
The granules used for the experiments are white monosized plastic beads of spherical shape having the following properties: diameter $d=5.85 \mathrm{~mm}$; density $=1.915 \mathrm{~g} / \mathrm{cm}^{3}$; angle of repose $=22.5$ degrees. In the present work, all experiments were conducted with the same volume of particles placed evenly in the central compartment, rising to a depth of approximately $12 \mathrm{~cm}$. Experiments are conducted by driving the conveyor belt and observing the resulting granular flow. Confined within the compartment, the granular material is driven upslope by the moving conveyor belt and pulled downslope by gravity. Preliminary experiments conducted at various inclinations and belt speeds indicated that the granules tend to heap at the upper end of the channel for low inclinations and at the lower end of the channel for higher inclinations. There is a unique inclination at which the depth of the deforming layer is approximately uniform over the whole length of the channel. This occurs for an inclination of 15.6 degrees. This angle is not measurably affected by the belt velocity at least up to speeds of $20 \mathrm{~cm} / \mathrm{s}$. All experiments described in the present paper were obtained at this precise inclination where the flow is approximately steady uniform.

To characterise the flows, digital video footage is acquired using a TRV-30 Sony DV camera. The camera is positioned to capture a side view of the granular layer close the channel mid-length, where the motion is most nearly parallel. The viewing window is inclined with the channel and covers the full depth of the granular layer. Illumination is provided by two spotlights oriented at approximately 45 degrees. The camera operates at a frequency of $30 \mathrm{~Hz}$ in interlaced mode (odd and even rows correspond to staggered time instants), with an image resolution of 480 rows by 720 columns. Before processing, images are split into their odd and even rows in order to obtain instantaneous images at twice the frame rate $(60 \mathrm{~Hz})$ but half the vertical resolution. For each experimental test, the granular flow runs for more than a minute at the 

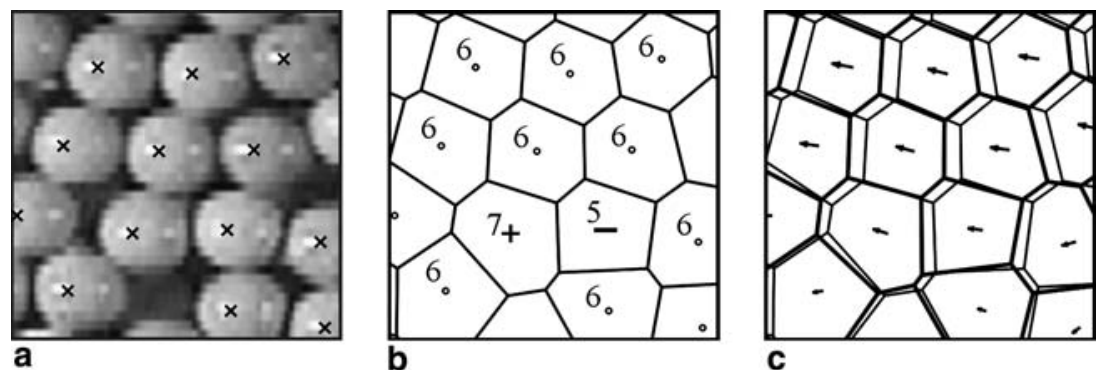

Fig. 2 Image analysis: a estimated particle positions; b Voronoï cells with counts of the number of edges of each cell; $\mathbf{c}$ particle displacements (arrows) extracted by matching Voronoï cell shapes between one image (thin lines) and the next (thick lines)

chosen conveyor belt speed before footage is acquired over a duration of 6 seconds.

\section{Image analysis}

We seek to analyse the configurations and motions of near-wall particles in the vertical $(x, y)$ plane (see Figure 1). Basic imaging algorithms (see reference [14] for details) are first used to automatically extract particle positions from the images (Figure 2a). De-interlaced frames are convoluted with a Laplacian-of-Gaussian filter. Particle centres are then identified as brightness maxima of the filtered images. Subpixel accuracy is obtained by fitting a quadratic surface to the neighbourhood of each maximum and taking the position of the surface peak as estimate of the particle position on the image. Calibrated scale factors are finally used to convert to physical units. This yields sets of coordinate pairs $\left(x_{i}^{(m)}, y_{i}^{(m)}\right)$, where a given pair denotes the position of the $i$-th particle identified on the $m$-th frame of the sequence.

The two subsequent steps both resort to the Voronoï diagram. For a given frame $m$, the Voronoï tiling assigns to each particle $i$ a polygonal region of the plane (its Voronoï cell) in which points lie closer to particle centre $\left(x_{i}, y_{i}\right)$ than to any other particle centre in the set. In the present work, we use the Voronoï diagram for two different purposes (Figure $2 \mathrm{~b}-\mathrm{c})$. The first purpose is to characterise the local configuration of near-wall grains on the basis of the number of sides of individual Voronoï cells [17]. Regions where particles are arrayed in a crystal-like packing feature 6-sided polygons only, while more disordered regions include polygons with different side counts, 5 or 7 sides being the most frequent (see Figure 2b). Such polygons appear in the neighbourhood of voids or dislocations in an otherwise hexagonal array, or in regions where particle arrangements are very irregular. Departures from crystalline packing can thus be quantified by introducing a local "defect density", defined as the ratio $v_{\delta}$ of the number of $n$-sided Voronoï cells $(n \neq 6)$ to the total number of Voronoï cells in a given region of the plane. To avoid edge effects, Voronoï cells located along the boundaries of the domain are excluded from consideration according to the criterion of reference [18].

The second task for which we resort to the Voronoï diagram is the velocimetric tracking of particles. Once sets of particle positions $\left(x_{i}^{(m)}, y_{i}^{(m)}\right)$ and $\left(x_{j}^{(m+1)}, y_{j}^{(m+1)}\right)$ have been identified on two successive images $m$ and $m+1$, the problem is to establish the one-to-one correspondence $j(i)$ linking two successive sightings of each physical particle. If this correspondence can be obtained, particle velocities $\left(u_{i}^{(m)}, v_{i}^{(m)}\right)$ can be approximated by [19]

$u_{i}^{(m)}=\frac{x_{j(i)}^{(m+1)}-x_{i}^{(m)}}{\Delta t}, \quad v_{i}^{(m)}=\frac{y_{j(i)}^{(m+1)}-y_{i}^{(m)}}{\Delta t}$.

In the present experiments, the particles are identical but move relatively slowly hence a reasonable correspondence $j$ (i) can be obtained by simply assuming that the next position of a given particle is the one closest to its previous position. This is the so-called minimum displacement algorithm [20]. To make this approach more robust, however, we also exploit point-pattern information extracted from the Voronoï diagram: two particle positions are considered good matches if the associated displacement is small and if their corresponding Voronoï cells are similar in shape, according to the criterion detailed in reference [14]. This is illustrated on Figure 2c.

Large sets of data are generated by the above imaging procedures. For each experimental run, some 250,000 Voronoï cell side counts and velocity vectors are obtained. The corresponding measurement errors depend on the pixel resolution of the images. Error analyses carried out in references [21, 22] indicate that typical root-mean-squared errors on position lie in the range $\varepsilon_{x} \approx 0.1$ to 0.25 pixel. In physical units, this gives position errors of the order of $\varepsilon_{x} \approx 0.03$ to $0.08 \mathrm{~mm}$ for the present experiments. Assuming that position errors are uncorrelated from one image to the next, the corresponding root-mean-squared error on velocity is

$\varepsilon_{v}=\sqrt{2} \frac{\varepsilon_{x}}{\Delta t} \approx 0.3$ to $0.7 \mathrm{~cm} / \mathrm{s}$.

The effect of these errors on the velocity fluctuation measurements will be examined below.

\section{Structural features}

Before examining defect distributions and velocities in a quantitative fashion, it is useful to take a qualitative look at a typical experimental run. We choose for this purpose the run obtained for conveyor belt speed $u_{0}=14.7 \mathrm{~cm} / \mathrm{s}$. Figures 3 and 4 show short-exposure and long-exposure views, 

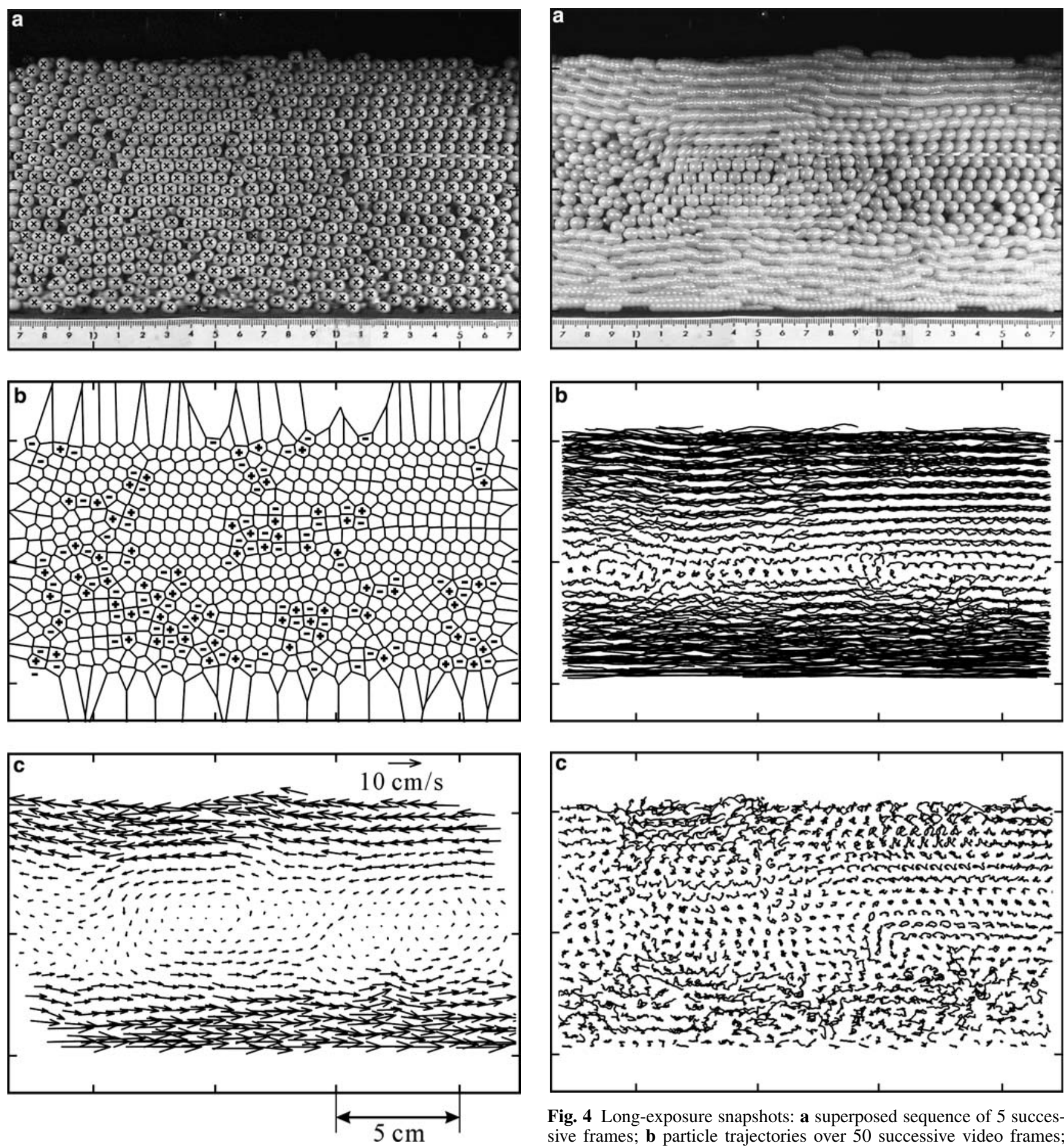

Fig. 4 Long-exposure snapshots: a superposed sequence of 5 successive frames; b particle trajectories over 50 successive video frames; c 'convected' particle trajectories (upon subtraction of the local mean flow component) over 50 successive video frames

Fig. 3 Short-exposure snapshots: a single video frame with marked particle positions $(\times)$; $\mathbf{b} n$-sided cells of the Voronoï diagram with indications of defects (+ for $n>6$ and - for $n<6$ ); c velocity vectors based on displacements over 5 successive frames

respectively. The figures depict a number of structural features similar to those documented in detail by Drake [11] in experiments with granular monolayers held between vertical parallel plates.

Panels $\mathrm{a}$ and $\mathrm{b}$ of Figure 3 document typical instantaneous configurations of near-wall particles during the slow

shear flow. Throughout the layer, particles are densely packed and interact with their neighbours through enduring contacts rather than short-lived collisional encounters. Only at the very top do some particles occasionally tumble downslope, bouncing rapidly along the free surface. Comparison of panels a and $\mathrm{b}$ illustrates the relationship between granular arrangements and the number of sides of their Voronoï cells. Side counts 
differing from 6 are clearly associated with localised defects or disordered regions. Conversely, 6-sided cells do not imply a perfect tiling of regular hexagons: distorted lattices also qualify, and regions where patterns are most irregular nonetheless yield a certain proportion of 6-sided cells.

Contrasted patterns are observed in the lower and upper parts of the flow. The lower part features disordered arrangements, as grains must adjust to the geometrically rough boundary of the conveyor belt. The trapezoidal grooves of the belt are periodic, but their dimensions do not match those of the grains, hence a disrupting effect felt up to some 5-10 diameters above the belt. In the upper part of the flow, on the other hand, one observes coherent blocks where particles are arrayed with crystal-like regularity. Two types of boundaries between blocks can be distinguished: clean fault lines oriented parallel to the bed or at angles of \pm 60 degrees, and irregular interstitial zones where defects tend to cluster.

Complementing the pattern information, panel $\mathrm{c}$ of Figure 3 shows the corresponding velocity field. The velocities shown are derived from individual particle displacements measured over 5 successive frames, and no spatial filtering or averaging has been applied. Superimposed upon the overall shear deformation, coherent granular motions are observed. Vortex-like structures similar to those noted in references [23, 24] are apparent at mid-depth. Comparison with panels a and $\mathrm{b}$ indicates that these correspond to rotations of the crystallike blocks. Zones across which velocities change directions further coincide with the boundaries between blocks. The flow field is approximately divergence-free, indicating that the compact granular flow behaves much like an incompressible phase. No slip is observed at the base of the flow: grain velocities at the base are equal to the speed of the conveyor belt $(14.7 \mathrm{~cm} / \mathrm{s}$ for this run).

Granular motions are further documented on Figure 4. Panel a is an artificial long exposure image obtained by merging together a sequence of 5 video frames. The image conveys an intuitive sense of how the flow deforms and corroborates the more precise information of Figure 3c. Panels $b$ and $c$ of Figure 4 are obtained by tracking particles over 50 successive frames, and indicate how particles move over a longer time interval $(0.83 \mathrm{sec})$. Particle trajectories are plotted in two different ways. Panel $\mathrm{b}$ shows the raw trajectories $(x(t), y(t))$, while panel c shows convected trajectories $\left(x^{\prime}(t), y^{\prime}(t)\right)$ obtained by subtracting a time integral of the local mean flow velocity from the raw trajectories. This amounts to observing individual particle paths in a frame of reference moving with the local mean flow, as suggested in reference [25].

Both sets of trajectories further highlight the internal structure of the slow granular flow. On panel $4 b$, contrasted pictures are again obtained for the lower and upper parts of the flowing layer. In the lower part, trajectories tend to distribute evenly over depth due to the more irregular arrangement of the corresponding grains. In the upper part, by contrast, trajectories cluster into horizontal threads, much like lanes of a highway along which cars follow one another, changing lanes only occasionally. Distinct horizontal rows of particles slide one on top of the other to accommodate the overall shear deformation. On panel $4 \mathrm{c}$, the convected trajectories highlight the block structure of the flow. In a given block, the motion histories of individual particles exhibit long range correlations, tracing orbits which are strikingly similar in shape. On the other hand different blocks exhibit dissimilar fluctuation velocity signatures. The motions of distinct blocks are not synchronised, and they move intermittently with respect to each other. These kinematical features closely match the "grain-layer gliding" and "block gliding" mechanisms described by Drake [11]. Layering of particles in organised rows has also been observed in computations of dense granular flows in the collisional regime [26], and in liquid-granular flow experiments [22].

\section{Defect density profiles}

For positions distributed in the 2D plane according to a completely random Poisson process, the most likely number of sides for a Voronoï cell is 6 . Side counts $n=4,5,7$ and 8 are also frequent, however, hence 6-sided cells occur with a relatively low probability frequency $P(n=6)=0.2946$ (see reference [27]). In the present granular flow experiments, the side count distribution is much narrower. Inside the flow, cells with 6 sides occur with a very high frequency $P(n=6) \approx 0.6$ to 0.9 . The remainder is mostly accounted for by 5 - and 7 sided cells. The proportions of these defects are nearly equal because they tend to occur in pairs (see Figure 3b). Very few cells have counts smaller than 5 or greater than 7 . The randomness of the granular arrangement is thus rather limited, as could be expected for densely packed, monosized particles which constrain each other through excluded volume effects.

To characterise the distribution of these defects over depth, defect density profiles are constructed in the following way. The flow layer is subdivided into 20 non-overlapping bins of constant thickness, into which individual measurements are distributed. Particle centres falling into each bin have the number of sides $n$ of their Voronoï cells counted. Cells for which $n \neq 6$ are recorded as lattice defects and contribute to the defect density of the sublayer. For each depth $y_{k}$, the defect density $v_{\delta}\left(y_{k}\right)$ is the ratio $v_{\delta}$ of the number of $n$-sided Voronoï cells $(n \neq 6)$ to the total number of Voronoï cells centred in the sublayer. The chosen bin thickness $\Delta y$ corresponds approximately to one grain diameter.

Depth profiles of the defect density $v_{\delta}\left(y_{k}\right)$ for the 14 different experimental runs are shown on Figure 5. Hollow symbols of different shapes correspond to separate runs obtained for different belt speeds (see Figure 7 below). As measured by indicator $v_{\delta}$, defects are observed to be most prevalent at the bottom and at the top of the flowing layer. Conversely, the most regular granular arrangements (low defect density $v_{\delta}$ ) are encountered in the middle of the layer. For any given run, a relatively regular $\mathrm{C}$-shaped curve is obtained for the distribution $v_{\delta}\left(y_{k}\right)$ of the defect density over depth.

Although they share a common shape, the curves obtained for different belt speeds present a substantial degree of scatter, 


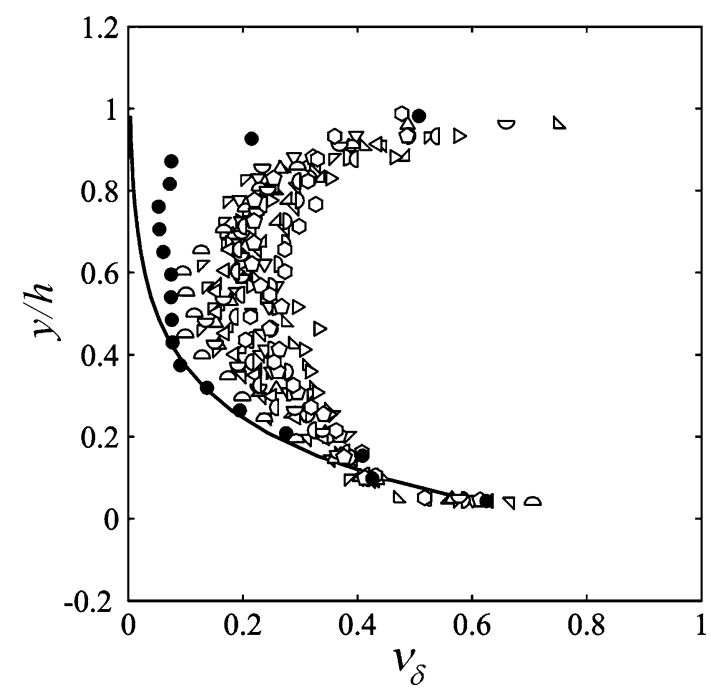

Fig. 5 Depth profiles of the defect density $v_{\delta}(y)$ for granular layers under shear (hollow symbols) and at rest (filled circles and exponential fit to the lower half of the data)

observed to be widest at mid-depth. Yet the defect density there does not vary with belt speed in any systematic fashion. Overall, no trend emerges which would suggest either a greater or lesser prevalence of defects as the belt speed rises, indicating that the defect density is not strongly dependent on the mean shear rate. Alternatively, the duration over which profiles are averaged may not be sufficient for convergence towards the long term mean, obscuring a relationship which may nonetheless be present. Since the velocity profiles do converge over the same time period of $6 \mathrm{~s}$ (see next section), the fact that regression to the mean is not observed for the defect density profiles suggests that two time scales may be present. Velocities would appear to adjust on a fast time scale, while geometrical disruptions of the particle packing may take a long time to evolve.

To clarify the above picture, a separate series of tests was conducted to measure the defect density profile $v_{\delta}\left(y_{k}\right)$ for granular layers at rest. For each test, steady uniform flow is first driven by the conveyor belt for at least one minute. The belt is then stopped, granular motion ceases completely, and a digital image of the static layer is acquired. In total, 32 still frames were acquired in this fashion. The defect density profile obtained by averaging the corresponding data is plotted as well on Figure 5. In the upper part of the flow, the static profile (filled disks) differs significantly from the dynamic profiles (hollow symbols). This indicates that even if no strong dependence is observed with respect to the rate of deformation, the granular assembly is nonetheless sensitive to being at rest or undergoing shear. While the static and dynamic data diverge in the upper part of the flow, they differ much less in the lower part. Defects there are very likely due to the geometrically rough boundary, which disrupts the granular packing regardless of whether the grains are moving or not. Seeking to separate this static boundary disruption from dynamic features possibly generated by the shear flow, we fit an exponential curve to the lower part of the static data (see Figure 5) and subtract the resulting profile from the dynamic data. The resulting defect density profiles are shown on Figure 6. Upon subtraction of the static fit, the defect density now monotonously decreases from a maximum at the free surface to a minimum at the bottom.

Inspired loosely from the void propagation description of reference [28], we can formulate a simple model which roughly accounts for the shape of the defect density profile. Assume that the sheared layer is subdivided into discrete rows of particles numbered (from bottom to top) $k=1, \cdots, N$ where $N=20 \approx h / d$ and $d$ is the particle diameter. The number density of defects in each row is $v_{\delta}[k]$ and is assumed to evolve according to the following non-dimensional, semidiscrete evolution equation

$$
\frac{\partial v_{\delta}[k]}{\partial \tau}=J\left[k-\frac{1}{2}\right]-J\left[k+\frac{1}{2}\right]+C
$$

where $\tau=t \dot{\gamma}$ is a dimensionless time set by the shear rate $\dot{\gamma}=|\partial \bar{u} / \partial z|$ (assumed uniform over the depth), $J\left[k+\frac{1}{2}\right]$ denotes a rate of transfer of defects from row $k$ to row $k+1$, and $C$ is the rate at which defects are generated (assumed constant throughout the layer). The transfer rate $J$ is assumed to take the form

$$
J\left[k+\frac{1}{2}\right]=A v_{\delta}[k]+B\left(v_{\delta}[k]-v_{\delta}[k+1]\right)
$$

where the first component is the rate at which defects migrate upwards (e.g. when a vacancy in a lower layer is filled by a grain from an upper layer, hence an upwards migration of the vacancy) and the second component is a diffusive flux. This flux formulation can be seen as a discrete analogue of the Rouse theory of suspended sediment transport [29]. Parameters $A$ and $B$ are again taken to be constant. Boundary conditions can finally be set as

$J\left[\frac{1}{2}\right]=0, \quad v_{\delta}[N]=0.7054$

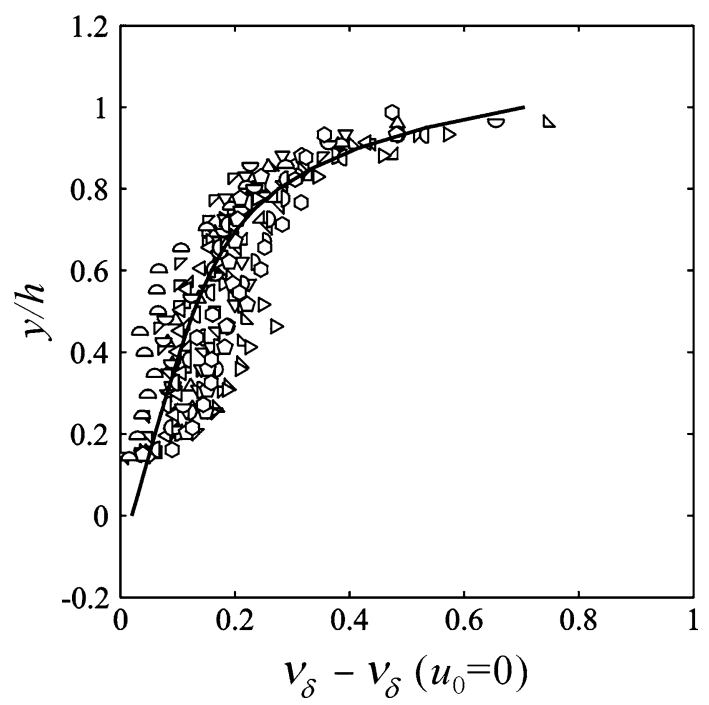

Fig. 6 Depth profiles of the defect density after subtraction of the static fit, $v_{\delta}-v_{\delta}\left(u_{0}=0\right)$ (hollow symbols) and rough $\mathrm{O} \Delta \mathrm{E}$ model fitted to the data (curve) 
i.e. no flux at the rigid bottom and maximum randomness (the value $v_{\delta}=1-P(n=6)$ corresponding to a Poisson point process) at the free surface. At steady state, $\partial v_{\delta}[k] / \partial \tau=0$ and the resulting ordinary difference equation $(\mathrm{O} \Delta \mathrm{E})$ can be solved (see e.g. [30]) to obtain

$$
\begin{aligned}
v_{\delta}[k]=\frac{B}{A} & \frac{C}{A}+\frac{C}{A} k+\left(0.7054-\frac{B}{A} \frac{C}{A}-\frac{C}{A} N\right) \\
& \times\left(\frac{B}{A+B}\right)^{N-k} .
\end{aligned}
$$

The steady profiles depend solely on the two ratios $B / A$ and $C / A$, and a reasonable fit to the measured data is obtained by choosing $B / A=2$ and $C / A=0.01$ (see Figure 6). The quality of the fit deteriorates significantly if any component of the above model is neglected.

This very rough model suggests that, once static effects have been discounted, the distribution of defects results from a dynamic balance between three processes: the downwards diffusion of defects starting from the more random free surface, the upwards migration of defects as a result of their buoyancy (the pull of gravity favors packings of greater density and regularity), and the ongoing generation of defects inside the bulk due to shear. In the above model, the shear rate influences the time scale on which these mechanisms act but not the shape of the equilibrium reached at steady state. The model clearly falls far short of accounting for the rich internal structure documented earlier (Figures 3 and 4), in particular the organisation of the grains into blocks. Nevertheless, it appears to account for some basic features of the distribution of defects over depth.

\section{Velocity profiles}

Like the particle configurations, the granular motions can be examined quantitatively. For this purpose, velocity profiles are constructed using the same subdivision of the flowing layer into non-overlapping bins. The average longitudinal velocity $\bar{u}\left(y_{k}\right)$ within the $k$-th bin is given by [10]

$\bar{u}\left(y_{k}\right)=\frac{\sum_{m} \sum_{i} I_{i}^{(m)}(k) u_{i}^{(m)}}{\sum_{m} \sum_{i} I_{i}^{(m)}(k)}$,

where $y_{k}$ is the height of the centre of the $k$-th bin and $I_{i}^{(m)}(k)$ is an indicator function defined by

$\begin{cases}I_{i}^{(m)}(k)=1 \text { if } y_{k}-\Delta y / 2 \leq y_{i}^{(m)}<y_{k}+\Delta y / 2 \\ I_{i}^{(m)}(k)=0 & \text { otherwise. }\end{cases}$

Once bin-averaged velocities $\bar{u}_{k}$ have been estimated, fluctuation velocities are taken as

$u_{i}^{\prime(m)}=u_{i}^{(m)}-\bar{u}\left(y_{i}^{(m)}\right)$,

where $\bar{u}\left(y_{i}^{(m)}\right)$ is obtained at the actual instantaneous position of the particle $y_{i}^{(m)}$ by linearly interpolating from profile $\bar{u}\left(y_{k}\right)$. Root-mean-squared (rms) velocity fluctuations in each bin are then given by

$\sqrt{\overline{u^{\prime 2}}}\left(y_{k}\right)=\sqrt{\frac{\sum_{m} \sum_{i} I_{i}^{(m)}(k) u_{i}^{\prime(m) 2}}{\sum_{m} \sum_{i} I_{i}^{(m)}(k)}}$

Depth-profiles of fluctuation velocities in the $y$-direction $\sqrt{\bar{v}^{\prime 2}}$ $\left(y_{k}\right)$ are obtained likewise.

Profiles were obtained for a series of 14 experimental runs conducted at belt speeds ranging from 4 to $18 \mathrm{~cm} / \mathrm{s}$. Figures 7 and 8 show the corresponding results for the mean and fluctuation velocities. On panel 7a, the mean longitudinal velocity profiles $\bar{u}(y)$ are plotted in dimensional units. On panel $7 \mathrm{~b}$, the same data are plotted in terms of dimensionless ratios $y / h$ and $\bar{u} / u_{0}$, where $h=12 \mathrm{~cm}$ is the approximate depth of the granular layer and $u_{0}$ is the speed of the conveyor belt (shown for each run as a filled symbol on panel 7a). Mean granular velocities at the base of the layer are practically equal to the belt speed, confirming previous observations that the no slip condition applies to the geometrically rough boundary.

Integration of the mean velocity profiles over depth yields values close to zero, as expected for a steady granular flow confined within rigid boundaries at the left and right sides
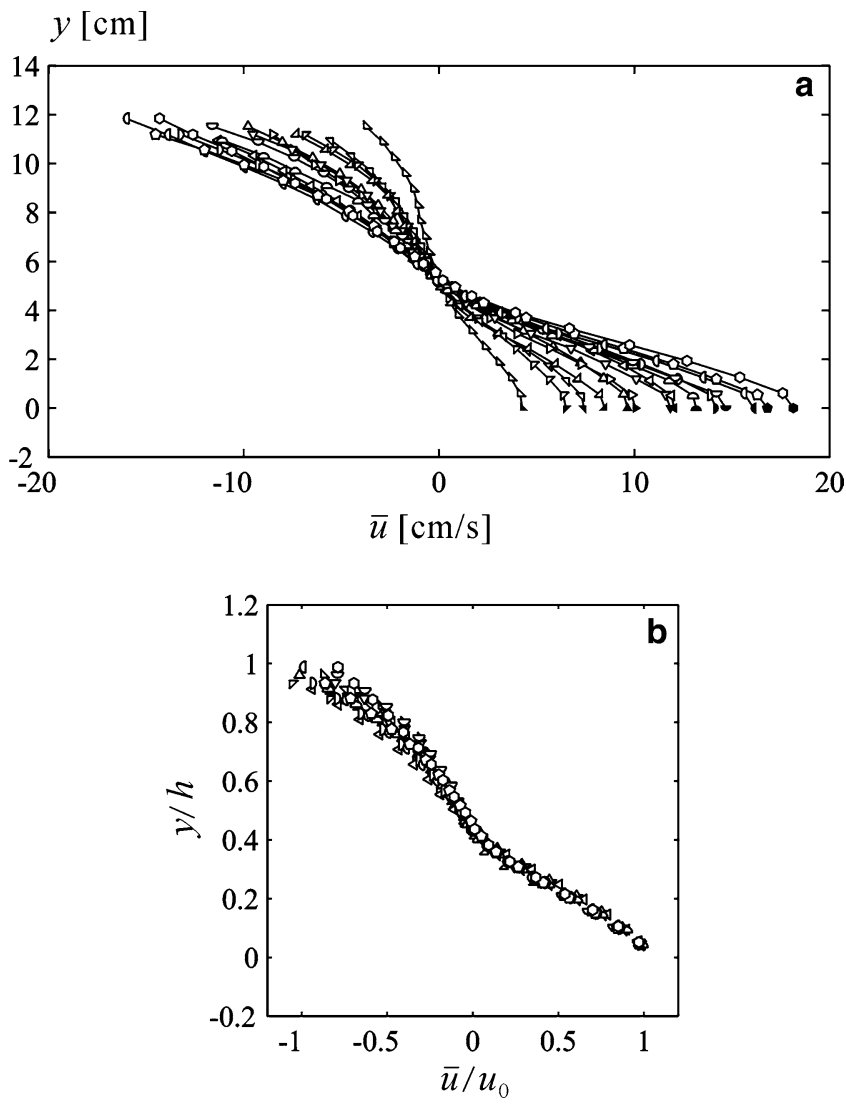

Fig. 7 Measured mean velocities $\bar{u}(y)$ for different conveyor belt speeds: a profile data (hollow symbols) in dimensional units, with belt speeds shown as filled symbol at the base of the layer; $\mathbf{b}$ data scaled with respect to depth $h$ and belt speed $u_{0}$ 

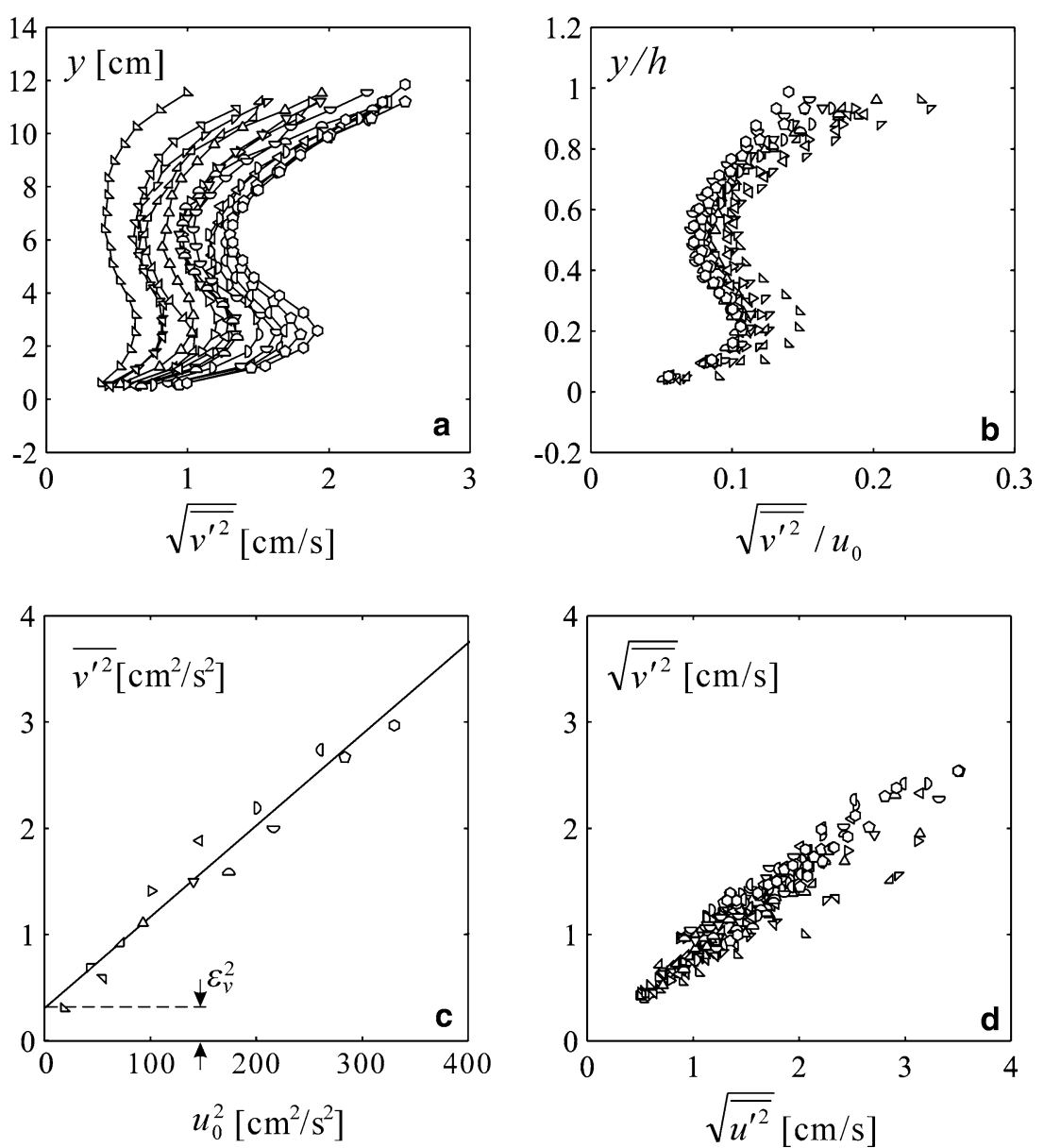

Fig. 8 Fluctuation velocities : a depth profile of normal-to-bed rms fluctuation velocity $\sqrt{\overline{v^{\prime 2}}}(y)$; $\mathbf{b}$ data scaled with respect to depth $h$ and belt speed $u_{0}$; $\mathbf{c}$ mean-squared fluctuation velocity $\overline{v^{2}}$ (averaged over the full depth) versus squared belt speed $u_{0}^{2}$; $\mathbf{d}$ comparison of longitudinal and normal-to-bed fluctuation velocities $\sqrt{\overline{u^{\prime 2}}}$ and $\sqrt{\overline{v^{\prime 2}}}$

of the channel. Grains move up along the bottom, driven by the belt, and flow back down along the surface. The profiles obtained for various belt speeds are highly similar in shape. For any given profile, the shear rate $\dot{\gamma}=|\partial \bar{u} / \partial y|$ peaks at the free surface, and decreases to a local minimum at mid-depth. The shear rate increases again in the lower half of the layer before decreasing in the immediate vicinity of the belt. The low shear rates observed at mid-depth most likely reflect the influence of friction along the sidewall. In that zone, mean velocities close to zero allow particles to undergo stick-slip motions, intermittently adhering to or sliding along the stationary wall. Where the mean velocity is further away from zero, by contrast, particles continuously slide along the wall.

As documented on panel $7 \mathrm{~b}$, the most striking feature of the flow is the way the profiles very nearly collapse together when the mean granular velocity is scaled with respect to the belt speed. This implies that the mean flow velocity throughout the layer is controlled by the speed of the conveyor belt. In other words, the self-similar profiles are kinematically constrained by the speed imposed at the lower boundary. The fact that steady uniform flow can be obtained for various belt speeds further implies rate-independent granular stresses, a hallmark of the slow frictional regime. In steady uniform flow, the shear and normal stresses at any given depth must balance the weight of the overlying granular layer, and are thus determined by the inclination of the channel and the packing density of the particles. Both these parameters are held virtually constant in the present experiments. Hence the stress levels at a given depth must be nearly identical for all runs. What varies is the rate of deformation, controlled by the speed of the conveyor belt as it increases from 4 to 18 $\mathrm{cm} / \mathrm{s}$. Thus stresses are not affected even when multiplying the deformation rate by a factor of 4 .

Fluctuation velocities are displayed on Figure 8. Panel 8a shows profiles of normal-to-bed rms fluctuation velocities $\sqrt{\overline{v^{\prime 2}}}(y)$ in dimensional units. Panel 8 b plots the same data in terms of dimensionless ratios $y / h$ and $\sqrt{\overline{\overline{v^{\prime 2}}}} / u_{0}$ where again the layer depth $h$ and conveyor belt speed $u_{0}$ are chosen as normalising variables. These profiles exhibit various features which are similar to those observed earlier for the mean velocity. First, fluctuation velocity profiles for different runs are highly similar in shape (panel 8a). Qualitatively, the fluctuation velocity evolves over depth in much the same 
way as the shear rate. For any given profile, the fluctuation velocity $\sqrt{\overline{v^{\prime 2}}}$ peaks at the free surface, decreases to a local minimum at mid-depth, then increases again in the lower half of the layer before decreasing in the immediate vicinity of the belt.

When scaled by the corresponding conveyor belt speeds, the fluctuation velocity measurements are also observed to approximately collapse together (panel 8b). The points do not align as closely as the mean velocity data (Figure 7), but this is likely due to limitations of the measurements rather than a physical effect. To check this inference, panel $6 \mathrm{c}$ plots the measured mean-squared vertical velocity fluctuations (averaged over the full depth) against the square of the belt speed. If the physical signal and the measurement noise are uncorrelated, the measured mean-square fluctuations will be offset by an additive contribution due to particle position errors, i.e.

$\left.\overline{v^{\prime 2}}\right|_{\text {measured }}=\left.\overline{v^{\prime 2}}\right|_{\text {true }}+\varepsilon_{v}^{2}$.

Assuming the true rms fluctuations to be proportional to the belt speed $u_{0}$, it follows that

$\left.\overline{v^{\prime 2}}\right|_{\text {measured }}=S u_{0}^{2}+\varepsilon_{v}^{2}$

where $S$ is the slope and $\varepsilon_{v}^{2}$ is the intercept of a linear relation. The measurements plotted on Figure 6c are seen to conform to this expectation. A linear fit yields for the square root of the intercept the value $\varepsilon_{v}=0.55 \mathrm{~cm} / \mathrm{s}$, which lies in the range of 0.3 to $0.7 \mathrm{~cm} / \mathrm{s}$ anticipated in section 3 for the velocity errors.

Finally, Fig. 8d compares normal-to-bed rms fluctuation velocities $\sqrt{\overline{v^{\prime 2}}}$ against the corresponding longitudinal fluctuations $\sqrt{\overline{u^{\prime 2}}}$ (taken at the same depth). On this panel, the fluctuation velocities in the two perpendicular directions $\sqrt{\overline{u^{\prime 2}}}$ and $\sqrt{\overline{v^{\prime 2}}}$ are seen to have roughly the same strength. A slight anisotropy is recorded, with longitudinal fluctuations $\sqrt{\overline{u^{\prime 2}}}$ stronger than normal-to-bed fluctuations $\sqrt{\overline{v^{\prime 2}}}$ by some $15 \%$. This feature is shared with simulations of collisional granular flows [31], although the rate-independence of the present observations puts them squarely in the frictional regime.

\section{Velocity correlations}

The previous section looked at the magnitudes of the mean and fluctuation components of the granular velocities. To further probe the nature of the velocity fluctuations, the present section examines their correlation signatures. Here we limit our data analysis to the experimental run (belt speed $\left.u_{0}=14.7 \mathrm{~cm} / \mathrm{s}\right)$ used earlier to illustrate the structural features of the flow (see section 4 as well as Figures 3 and 4). Both time and space correlations are of interest, and we examine them in sequence.

The particle tracking techniques used in the present work make it possible to follow the individual trajectories of particles over many successive frames (see Figure 4). This in turn allows Lagrangian velocity autocorrelation functions to be determined. Following ref. [25], scaled correlations of the fluctuating particle velocities are defined as:

$$
\begin{aligned}
& R_{L x x}(\delta t)=\frac{\overline{u^{\prime}(t) u^{\prime}(t+\delta t)}}{\overline{u^{\prime 2}}}, R_{L x y}(\delta t)=\frac{\overline{u^{\prime}(t) v^{\prime}(t+\delta t)}}{\sqrt{\overline{u^{\prime 2}}} \sqrt{\overline{v^{\prime 2}}}} \\
& R_{L y x}(\delta t)=\frac{\overline{v^{\prime}(t) u^{\prime}(t+\delta t)}}{\sqrt{\overline{v^{\prime 2}}} \sqrt{\overline{u^{\prime 2}}}}, R_{L y y}(\delta t)=\frac{\overline{v^{\prime}(t) v^{\prime}(t+\delta t)}}{\overline{v^{\prime 2}}}
\end{aligned}
$$

and characterise the correlation between the $x$ - and $y$-components of the velocity fluctuations, along the trajectory of a given particle, at two different instants separated by elapsed time $\delta t$. Correlations involving the normal-to-wall component $w^{\prime}$ are not included because they cannot be derived from our 2D near-wall observations. For rapid granular flows at low and moderate solid fractions, Lagrangian autocorrelation functions have been found by various investigators ([25, $26,32]$ ) to follow exponentially decaying curves. This reflects the memory loss experienced by particles as they randomly collide with their neighbours.

The present experiments make it possible to probe instead slow, dense flows. Using the particle tracking measurements described earlier, the Lagrangian time correlation functions (13) can be estimated at various depths. For each depth, data from various trajectories are pooled together on the basis of the mean $y$ position of each trajectory, using the same binning sublayers as before. The time separation $\delta t$ is restricted to multiples of the sampling time $\Delta t=1 / 60 \mathrm{~s}$. Due to video interlacing, a noise component of frequency $60 \mathrm{~Hz}$ perturbs the $y$-components of velocity, and is eliminated using a binomial filter of mask [ $\left.\begin{array}{lll}1 & 2 & 1\end{array}\right] / 4$.

Results for two different sublayers of our reference run are shown on Figure 9. Panels a and b correspond to depths $y / h=2 / 3$ (near the top free surface) and $y / h=1 / 3$ (near the bottom conveyor belt), respectively. The time elapsed $\delta t$ is normalised using the mean shear rate $\overline{\dot{\gamma}}$ (averaged over the entire flow depth). For both sublayers, exponential decay is observed close to the origin for the $x x$ and $y y$ correlation functions. Away from the origin, however, the remaining correlations present a well-defined periodic component. Very similar oscillatory tails have been observed earlier in computational simulations of high-density granular flows [25], where they were ascribed to coordinated particle movements within an ordered microstructure. As shown on Figures 3 and 4, this appears to fit the present case. In the present experiments, particle configurations present a high degree of order. One would expect, for instance, that the motion of a grain past a row of aligned particles would generate a periodic component in its velocity autocorrelation function.

Root-mean-squared fluctuations in the two perpendicular directions $\sqrt{\overline{u^{\prime 2}}}$ and $\sqrt{\overline{v^{\prime 2}}}$ were observed earlier to be roughly equivalent in strength (to within $15 \%$ ), and to vary only moderately over the flow depth. It can further be seen on Figure 9 that, although different from zero, $R_{L x y}(\delta t=0)$ is relatively small, implying that

$\overline{u^{\prime} v^{\prime}}<<\sqrt{\overline{u^{\prime 2}}} \sqrt{\overline{\overline{v^{\prime 2}}}}$. 

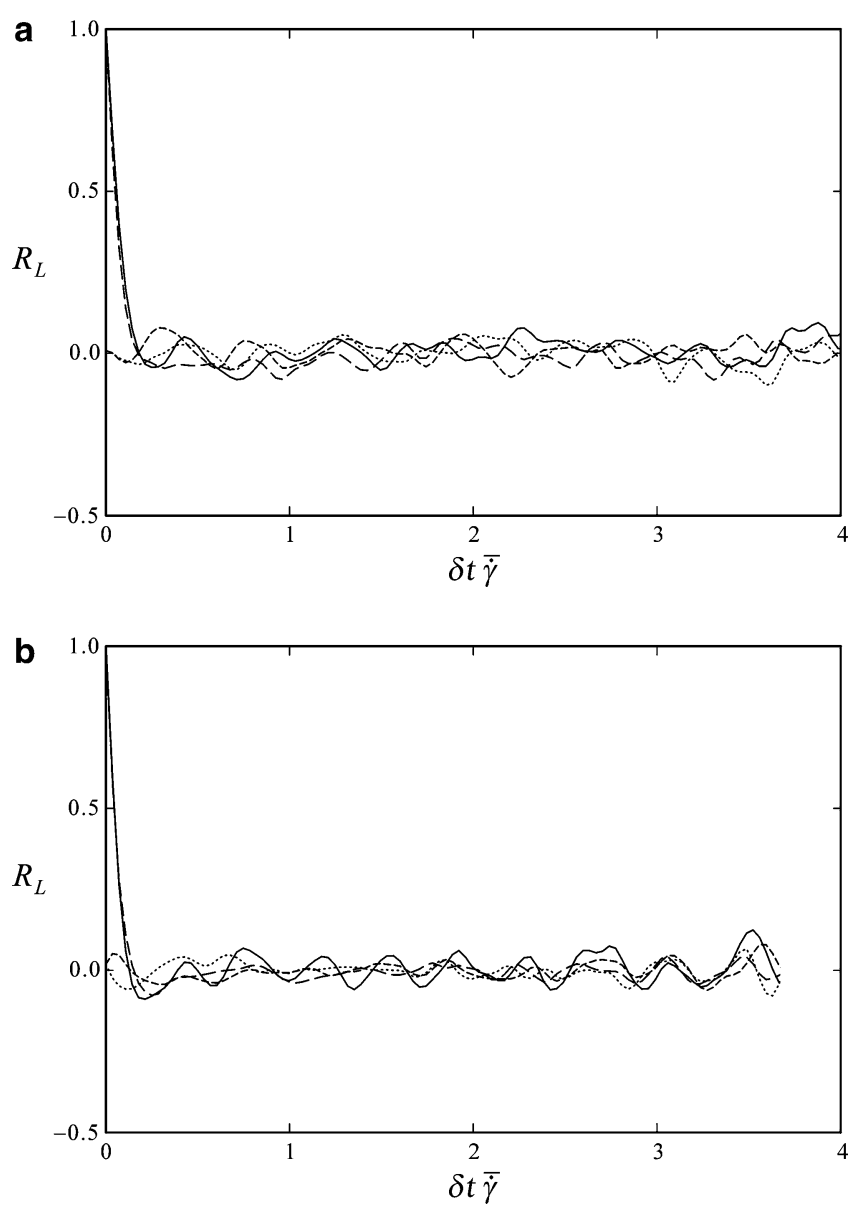

Fig. 9 Lagrangian velocity correlation functions $R_{L x x}(\delta t)$ (continuous line), $R_{L y y}(\delta t)$ (long dashes), $R_{L x y}(\delta t)$ (short dashes) and $R_{L x y}(\delta t)$ (dots), at two different depths: a $y / h=0.7 ; \mathbf{b} y / h=0.3$. Data for belt speed $u_{0}=14.7 \mathrm{~cm} / \mathrm{s}$

In other words, the correlation between the $x$ - and $y$-components of the fluctuating motions is weak. This contrasts with the situation encountered for dilute collisional granular shear flows and turbulent shear flows, in which these correlations are significant and make an important contribution to the shear stresses. Despite involving shear flows, the present experiments therefore appear to feature velocity fluctuations which are close to isotropic.

The above observations suggest that spatial correlations can be approached using simple tools borrowed from studies of homogoneous isotropic turbulence (see ref. [33]). For locally homogeneous and isotropic fluctuations, it is shown in [33] that the full spatial correlation structure can be reduced to two functions:

$$
\begin{aligned}
f_{\|}(\delta r) & =\frac{\overline{u_{\|}^{\prime}(\mathbf{r}) u_{\|}^{\prime}(\mathbf{r}+\delta \mathbf{r})}}{\sqrt{\overline{\overline{u^{\prime 2}}} \sqrt{\overline{v^{\prime}}}}} \quad \text { and } \\
f_{\perp}(\delta r) & =\frac{\frac{u_{\perp}^{\prime}(\mathbf{r}) u_{\perp}^{\prime}(\mathbf{r}+\delta \mathbf{r})}{\sqrt{\overline{u^{\prime 2}}} \sqrt{\overline{v^{\prime 2}}}}}{}
\end{aligned}
$$

where $\delta \mathbf{r}$ is the relative position vector, and $\delta r=\|\delta \mathbf{r}\|$ is the corresponding interdistance. Velocities $u_{\|}^{\prime}$ and $u_{\perp}^{\prime}$ are parallel and transverse fluctuation velocities defined as projections of the fluctuation velocity vector $\mathbf{u}^{\prime}$ onto direction $\delta \mathbf{r}$ and a direction perpendicular to $\delta \mathbf{r}$, respectively. For $3 \mathrm{D}$ incompressible flows, continuity requires that [33]

$$
f_{\perp}(\delta r)=f_{\|}(\delta r)+\frac{1}{2} \delta r \frac{\partial f_{\|}}{\partial(\delta r)},
$$

a relation that can be used to test if the granular medium deforms like an incompressible fluid.

For the present near-wall observations of dense granular flows, vector $\delta \mathbf{r}$ has components $\delta \mathbf{r} \approx(\delta x, \delta y, 0)$ (all viewed particles approximately lie in the same plane, parallel to the sidewall and one radius away from it). In that case, we have

$\delta r=\sqrt{(\delta x)^{2}+(\delta y)^{2}}, \quad u_{\|}^{\prime}=\frac{1}{\delta r}\left(u^{\prime} \delta x+v^{\prime} \delta y\right)$,

$u_{\perp}^{\prime}=\frac{1}{\delta r}\left(-u^{\prime} \delta y+v^{\prime} \delta x\right)$.

Using our particle tracking measurements, spatial correlation functions $f_{\|}(\delta r)$ and $f_{\perp}(\delta r)$ are estimated by averaging over particle pairs binned according to their interdistance $\delta r$. We evaluate the functions separately for different sublayers of the flow, on the basis of the normal-to-bed position $y$ of the
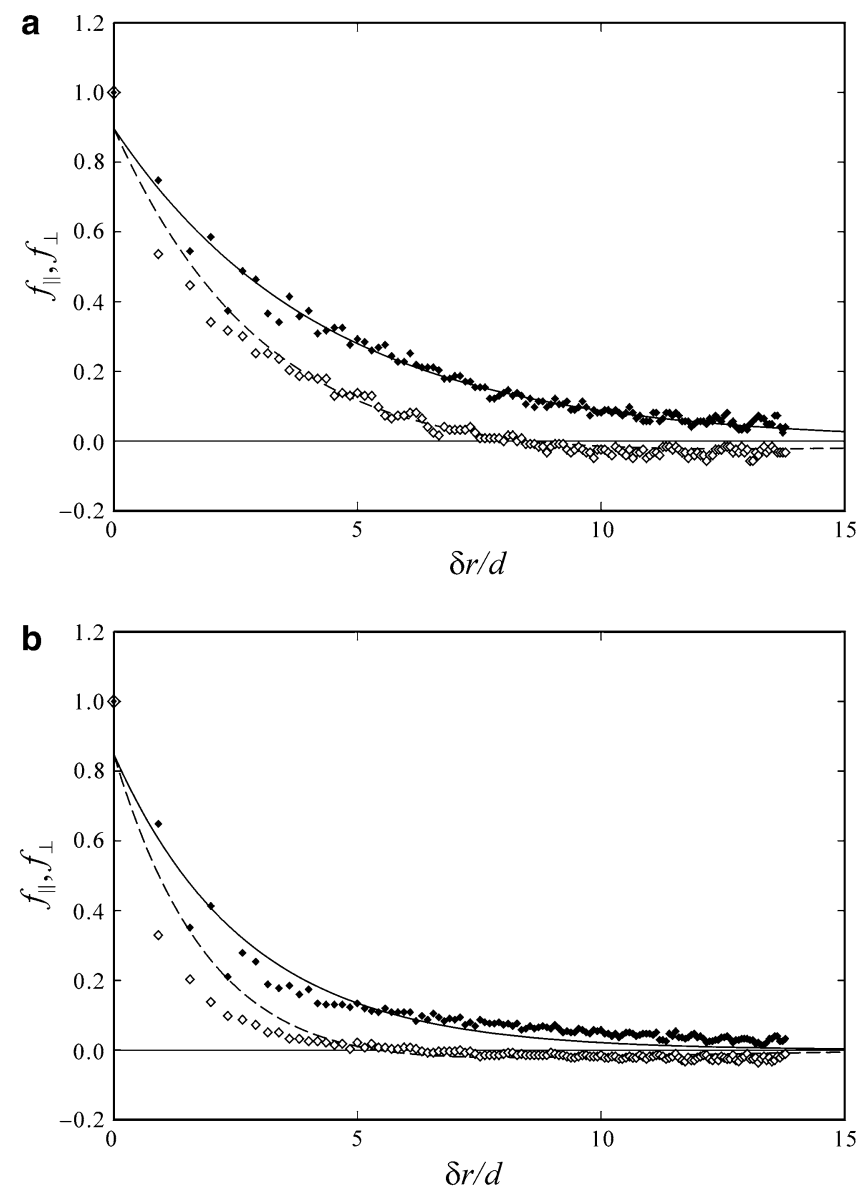

Fig. 10 Eulerian radial velocity correlation functions $f_{\|}(\delta r)$ (filled symbols $=$ data, continuous line $=$ exponential fit $)$ and $f_{\perp}(\delta r)$ (hollow symbols, dashed line), at two different depths: a $y / h=0.7$; b $y / h=0.3$. Data for belt speed $u_{0}=14.7 \mathrm{~cm} / \mathrm{s}$ 

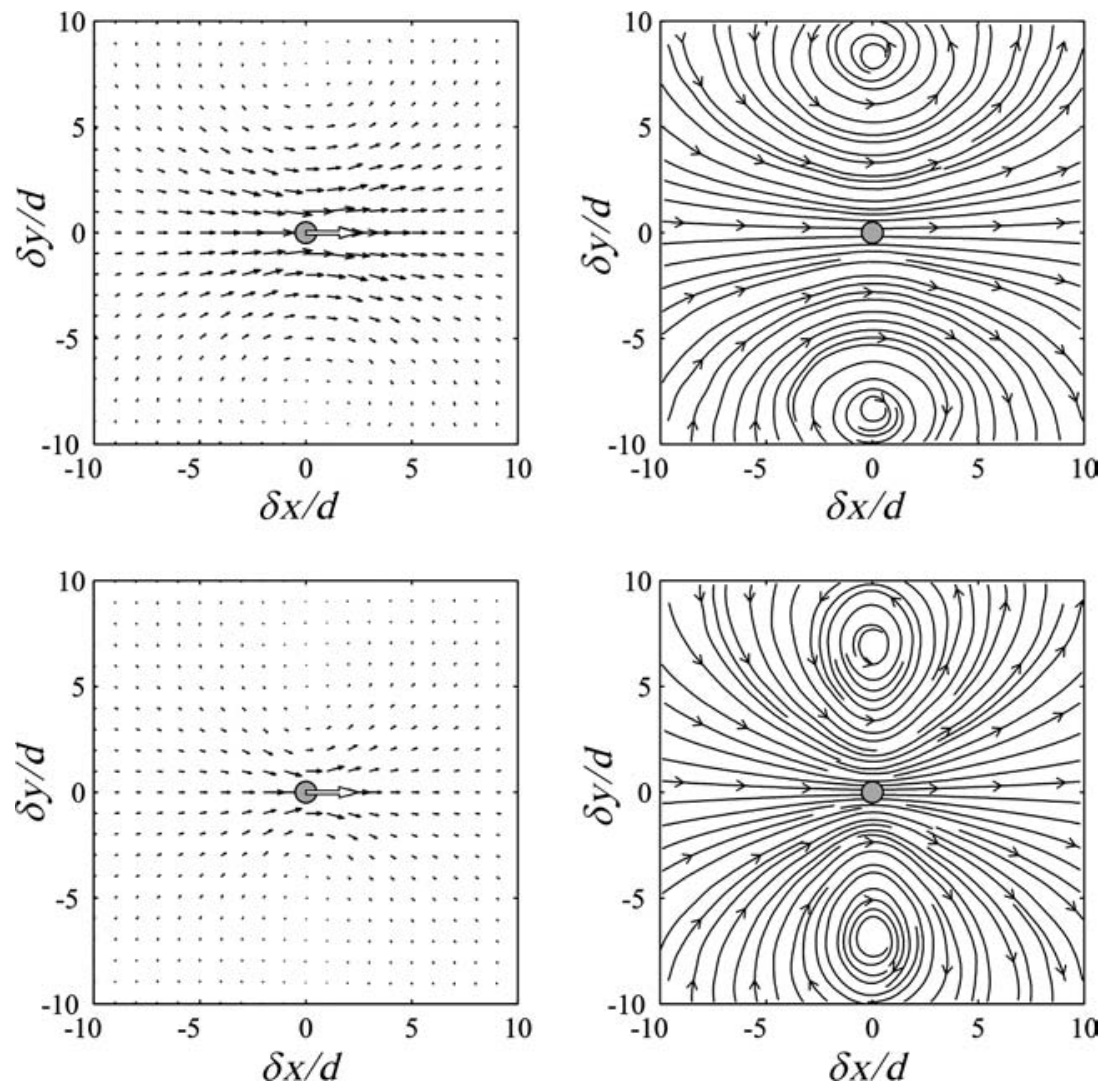

Fig. 11 Velocity field representation of the radial correlation data of Fig. 10. Top: $y / h=0.7$; bottom: $y / h=0.3$; left: vectors; right: streamlines

first particle of the pair. These separate evaluations, which would not be necessary if the flow was truly homogeneous, are meant to detect possible variations of the spatial correlation signature over depth.

Figure 10 shows spatial correlation results for two sublayers of our reference run. As in Figure 9, panels a and b correspond to depths $y / h=2 / 3$ (near the top) and $y / h=1 / 3$ (near the bottom), respectively. On both plots, the results for the parallel and transverse components resemble the correlation curves measured in 3D fluid turbulence experiments (see [33]). The parallel correlation $f_{\|}$decays with interdistance $\delta r$ but never drops below zero. On the other hand, the transverse correlation $f_{\perp}$ presents a steeper drop and becomes negative for large values of $\delta r$. The parallel correlation data of panel a are well approximated by a least squares fit taking the simple exponential form $f_{\|}(\delta r)=A \exp \{-B \delta r\}$. Assuming incompressible flow, equation (16) then yields the corresponding transverse correlation function $f_{\perp}(\delta r)=(1-$ $\left.\frac{1}{2} B \delta r\right) f_{\|}(\delta r)$. The resulting curve is found to approximate the transverse correlation data quite well, which implies that the granular assembly behaves very nearly like an incompressible medium. Turning our attention to panel $\mathrm{b}$, the data for the lower sublayer are seen to be poorly approximated by an exponential fit. Rapid decay is observed for small interdistances $\delta r$, giving way to slow decay for large interdistances. Because the exponential fit is poor for the parallel component, the equally poor fit of the associated transverse function $f_{\perp}(\delta r)=\left(1-\frac{1}{2} B \delta r\right) f_{\|}(\delta r)$ does not yield much information.

Based on the functions $f_{\|}$and $f_{\perp}$, one can reconstruct the full velocity correlation tensor. In particular, correlations in the $2 \mathrm{D}$ vertical plane (corresponding to our near-wall observations) can be obtained from expressions

$$
\begin{gathered}
\frac{\overline{u^{\prime}(\mathbf{r}) u^{\prime}(\mathbf{r}+\delta \mathbf{r})}}{\sqrt{\overline{u^{\prime 2}}} \sqrt{\overline{v^{\prime 2}}}}=\left\{f_{\|}(\delta r)-f_{\perp}(\delta r)\right\} \\
\frac{(\delta x)^{2}}{(\delta x)^{2}+(\delta y)^{2}}+f_{\perp}(\delta r), \\
\frac{\overline{u^{\prime}(\mathbf{r}) v^{\prime}(\mathbf{r}+\delta \mathbf{r})}}{\sqrt{\overline{u^{\prime 2}}}} \sqrt{\overline{v^{\prime 2}}}=\left\{f_{\|}(\delta r)-f_{\perp}(\delta r)\right\} \frac{\delta x \delta y}{(\delta x)^{2}+(\delta y)^{2}} .
\end{gathered}
$$

(or equivalent expressions for the $\overline{v^{\prime} u^{\prime}}$ and $\overline{v^{\prime} v^{\prime}}$ components of the tensor). To gain a qualitative feel for the implied behaviour, it is useful to see expressions (18) and (19) as two components of a vector field in the $(\delta x, \delta y)$ plane. The measured spatial correlations can then be plotted like a standard velocity field. Proposed in the classical paper [34], such a representation is shown on the left hand side of Figure 11, with associated streamlines plotted on the right hand side of the 
same figure. It can be interpreted as follows: a particle moving to the right (central arrow on the plots) experiences a correlated movement of the surrounding particles according to the pattern shown. The backflow induced above and below the central particle some distance away can be traced to a feature of the correlation functions mentioned earlier (see Figure 10): the dip of the transverse correlation function $f_{\perp}(\delta r)$ below zero for large values of $\delta r$. The observed pattern resembles the flow field generated by a vortex ring.

Taken together, these observations suggest that the slow deformation of a dense granular medium is surprisingly similar to the turbulent flow of an incompressible fluid. Fluctuations are spatially correlated and approximately divergence free. The resulting vortex patterns are visible both on snapshots of the velocity field (Figure 3 ) and on representations of the velocity correlation tensor (Figure 11). As in recent computations of cooling granular fluids $[23,35]$, granular turbulence is observed rather than molecular chaos.

\section{Conclusions}

Before concluding, important limitations of the present work must again be acknowledged. The present observations of 3D flow are restricted to 2D projections of granular patterns and motions visible near the channel sidewall, monitored over a limited time. In addition, particle patterns were probed using but a single indicator of packing regularity. Finally, a limited range of flow conditions was explored, excluding for instance variations in depth of the granular layer or belt speeds fast enough to reach collisional flow conditions.

Various tentative conclusions can nonetheless be drawn. First, observations of the distribution of lattice defects over depth indicate that two types of effects can be distinguished: 1) static disruption by the geometrically rough boundary which affects nearby grains regardless of whether they are moving or not; 2) a dynamic balance of ordering and disordering mechanisms which intervenes when the granular layer is undergoing shear.

Secondly, the measurements of mean velocities underscore the high degree of rate-independence which constitutes the hallmark of the frictional regime. Profiles obtained over a range of conveyor belt speeds are strikingly self-similar, and collapse together when scaled with respect to the belt speed. Although the scatter is wider, velocity fluctuations are found to roughly scale with the belt speed as well. Variations of the belt speed further have no clear influence on the density of lattice defects. This suggests that the microscopic features of the flow, just like the macroscopic mean velocities, exhibit a certain degree of invariance with respect to the rate of deformation.

Thirdly, additional information is provided by the time and space correlations. Lagrangian time auto-correlation functions exhibit oscillatory tails, which appear to be due to the highly ordered microstructure of the flow. Most strikingly, the granular velocity fluctuations are found to be correlated in space over scales of 5 to 10 grain diameters. Comparison of the parallel and transverse components of the velocity correlation suggests that, despite the presence of voids and defects, the granular phase behaves much like an incompressible fluid. As a result, the coherent fluctuation velocities adopt vortex patterns which resemble turbulent fluid motions. These kinematic similarities are surprising because they arise despite clear differences in dynamics: inertia-dominated motions for fluid turbulence, against slow frictional deformations for the present granular flow experiments.

While the above observations may provide some useful indications, even a cursory look at Figures 3 and 4 makes it clear that they are far from accounting for the rich internal structure of slow granular flows. This structure includes a dual organisation of particles into longitudinal chains and extended blocks, inducing long range correlations of both particle motions and arrangements. Intermittency in the evolution of these features is another defining characteristic difficult to convey in still images but clearly apparent when looking at the videos. Further probing of slow granular flows is clearly needed if one hopes to elucidate the precise mechanisms underlying such phenomena.

Acknowledgements Financial support for the experimental equipment used in the present research was provided to H. T. Chou by the National Science Council, Taiwan, through grant no. 91-2625-Z-008-007. Partial support for the work of $\mathrm{H}$. Capart was provided by the National Science Council, Taiwan, via grant no. 90-2211-E-002-084.

\section{References}

1. Jenkins, J.T., Savage, S.B.: A theory for the rapid flow of identical, smooth, nearly elastic particles. J. Fluid Mech. 130, 186-202 (1983)

2. Azanza, E., Chevoir, F., Moucheront, P.: Experimental study of collisional granular flows down an inclined plane. J. Fluid Mech. 400, 199-227 (1999)

3. Zhang, Y., Campbell, C.S.: The interface between fluid-like and solid-like behavior in two-dimensional granular flows. J. Fluid Mech. 237, 541-568 (1992)

4. Aharonov, E., Sparks, D.: Rigidity phase transition in granular packings. Phys. Rev. E, 60(6), 6890-6896 (1999)

5. Savage, S.B.: Analyses of slow high-concentration flows of granular materials. J. Fluid Mech. 377, 1-26 (1998)

6. Pouliquen, O., Forterre, Y., Le Dizes, S.: Slow dense granular flows as a self-induced process. Adv. Complex Systems. 4(4), 441-450 (2001)

7. Davies, T.R.H.: Debris flow surges - a laboratory investigation. Mitteilungen der Versuchsanstalt für Wasserbau, Hydrologie und Glaziologie, Nr. 96, ETH Zürich, (1988)

8. Hübl, J., Steinwendtner, H.: Estimation of rheological properties of viscous debris flow using a belt conveyor. Phys. Chem. Earth B, 25(9), 751-755 (2000)

9. Jain, N., Ottino, J.M., Lueptow, R.M.: An experimental study of the flowing granular layer in a rotating tumbler. Phys. Fluids 14(2), 572-582 (2002)

10. Hsiau, S.S., Shieh, Y.M.: Fluctuations and self-diffusion of sheared granular material flows. J. Rheol. 43, 1049-1066 (1999)

11. Drake, T.G.: Structural features in granular flows. J. Geophys. Res. 95(B6), 8681-8696 (1990)

12. Okabe, A., Boots, B., Sugihara, K.: Spatial Tesselations: Concepts and Applications of Voronoï Diagrams. Wiley, (1992) 
13. Richard, P., Oger, L., Lemaître, J., Samson, L., Medvedev, N.N.: Application of the Voronoï tesselation to study transport and segregation of grains inside 2D and 3D packings of spheres. Gran. Matter 1, 203-211 (1999)

14. Capart, H., Young, D.L., Zech, Y.: Voronoï imaging methods for the measurement of granular flows. Exp. Fluids 32, 121-135 (2002)

15. Bonamy, D., Daviaud, F., Laurent, L., Mills, P.: Texture of granular surface flows: experimental investigation and biphasic non-local model. Gran. Matter 4, 183-190 (2003)

16. Spinewine, B., Capart, H., Larcher, M., Zech, Y.: Three-dimensional Voronoï imaging methods for the measurement of near-wall particulate flows. Exp. Fluids 34, 227-241 (2003)

17. Allen, M.P., Frenkel, D., Gignac, W.: A Monte Carlo simulation study of the two-dimensional melting mechanism. J. Chem. Phys. 78(6), Part II: 4206-4222 (1983)

18. Kenkel, N.C., Hoskins, J.A., Hoskins, W.D.: Edge effects in the use of area polygons to study competition. Ecology 70, 272-274 (1989)

19. Adrian, R.J.: Particle-imaging techniques for experimental fluid mechanics. Annu. Rev. Fluid Mech. 23, 261-304 (1991)

20. Guler, M., Edil, T.B., Bosscher, P.J.: Measurement of particle movement in granular soils using image analysis. J. Comp. Civ. Eng. 13(2), 116-122 (1999)

21. Veber, P., Dahl, J., Hermansson, R.: Study of the phenomena affecting the accuracy of a video-based Particle Tracking Velocimetry technique. Exp. Fluids 22, 482-488 (1997)

22. Armanini, A., Capart, H., Fraccarollo, L., Larcher, M.: Rheological stratification in experimental free-surface flows of granular-liquid mixtures. J. Fluid Mech. 532, 269-319 (2005)
23. van Noije, T.P.C., Ernst, M.H.: Cahn-Hilliard theory for unstable granular fluids. Phys. Rev. E, 61(2), 1765-1782 (2000)

24. Utter, B., Behringer, R.P.: Self-diffusion in dense granular shear flows. Phys. Rev. E, 69, 031308-1-12 (2004)

25. Campbell, C.S.: Self-diffusion in granular shear flows. J. Fluid Mech. 348, 85-101 (1997)

26. Savage, S.B., Dai, R.: Studies of granular shear flows. Wall slip velocities, 'layering' and self-diffusion. Mech. Mater. 16, 225-238 (1993)

27. Le Caer, G., Ho, J.S.: The Voronoï tesselation generated from eigenvalues of complex random matrixes. J. Phys. A, 23, 3279-3295 (1990)

28. Caram, H., Hong, D.C.: Random-walk approach to granular flows. Phys. Rev. Lett. 67(7), 828-831 (1991)

29. Rouse, H.: Modern conceptions of the mechanics of fluid turbulence. Trans. ASCE 102, 532-536 (1937)

30. Kelley, W.G., Peterson, A.C.: Difference equations. Harcourt Academic Press, (2001)

31. Campbell, C.S.: The stress tensor for simple shear flows of a granular material. J. Fluid Mech. 203, 449-473 (1989)

32. Wildman, R.D., Huntley, J.M., Hansen, J.-P.: Self-diffusion of grains in a two-dimensional vibrofluidized bed. Phys. Rev. E, 60(6), 7066-7075 (1999)

33. Batchelor, G.K.: The Theory of Homogeneous Turbulence. Cambridge Univ. Press, (1970)

34. Alder, B.J., Wainwright, T.E.: Decay of the velocity autocorrelation function. Phys. Rev. A, 1(1), 18-21 (1970)

35. Isobe, M.: Velocity statistics in two-dimensional granular turbulence. Phys. Rev. E, 68, 040301-1-4 (2003) 